12-1-1999

\title{
Three-dimensional Structure of the Gly121Tyr Dimeric Form of Ornithine Decarboxylase from Lactobacillus 30a
}

Jacqueline Vitali

Cleveland State University, j.vitali@csuohio.edu

Donald Carroll

University of Texas at Austin

Rochika G. Chaudhrya

University of Texas at Austin

Marvin L. Hackert

University of Texas at Austin

Follow this and additional works at: https://engagedscholarship.csuohio.edu/sciphysics_facpub

Part of the Biochemistry Commons

How does access to this work benefit you? Let us know!

Publisher's Statement

(C) International Union of Crystallography 1999

\section{Original Citation}

Vitali, J., Carroll, D., Gopal, R. and Hackert, M.L. (1999) The Three-dimensional Structure of the Gly121Tyr Dimeric Form of Ornithine Decarboxylase from Lactobacillus 30a. Acta Crystallographica D55, 1978-1985.

\section{Repository Citation}

Vitali, Jacqueline; Carroll, Donald; Chaudhrya, Rochika G.; and Hackert, Marvin L., "Three-dimensional Structure of the Gly121Tyr Dimeric Form of Ornithine Decarboxylase from Lactobacillus 30a" (1999). Physics Faculty Publications. 184.

https://engagedscholarship.csuohio.edu/sciphysics_facpub/184

This Article is brought to you for free and open access by the Physics Department at EngagedScholarship@CSU. It has been accepted for inclusion in Physics Faculty Publications by an authorized administrator of EngagedScholarship@CSU. For more information, please contact library.es@csuohio.edu. 
Acta Crystallographica Section D

Biological

Crystallography

ISSN 0907-4449

\section{Jacqueline Vitali, Donald Carroll, Rochika G. Chaudhryt and Marvin L. Hackert*}

Department of Chemistry and Biochemistry, University of Texas, Austin, TX 78712, USA

\author{
+ Present address: Pan American Health \\ Organization - WHO, 525 Twenty-Third Street, \\ Washington, DC 20037, USA.
}

Correspondence e-mail: m.hackert@mail.utexas.edu
C) 1999 International Union of Crystallography Printed in Denmark - all rights reserved

\section{Three-dimensional structure of the Gly121Tyr dimeric form of ornithine decarboxylase from Lactobacillus 30a}

Ornithine decarboxylases catalyze the conversion of ornithine to putrescine at the beginning of the polyamine pathway. Ornithine decarboxylase (ODC) from Lactobacillus 30a is a 990612 Da dodecamer composed of six homodimers. A single point mutation (Gly121Tyr) was found to prevent association of dimers into dodecamers. The dimeric protein has been crystallized at $\mathrm{pH} 7.0$ in the presence of guanosine triphosphate (GTP). Crystals belong to space group $P 3_{2} 21$, with unitcell parameters $a=111.8, c=135.9 \AA$ and one monomer in the asymmetric unit. The structure was determined by molecular replacement and refined using simulated annealing to $R=0.211$ at $2.7 \AA$ resolution. The GTP-binding site was analyzed in detail. The protein exhibits a novel binding mode for GTP which is different from that seen in most G-proteins or GTPases. Central to this binding scheme appear to be three lysines, Lys190, Lys374 and Lys382, which form salt bridges with the three phosphates, and Thr191, which hydrogen bonds with the guanine base. Furthermore, the structure suggests that there is some flexibility in the wing domain, which can change its orientation as the protein adapts to its environment. The active site is similar to that of the native enzyme, consistent with the observation that the enzyme activity does not depend on its dodecameric state.

\section{Abbreviations}

AAT, aspartate aminotransferase; ADC, arginine decarboxylase; ALR, alanine racemase; DEAE, diethylaminoethyl; DTT, dithiothreitol; EDTA, $N, N, N^{\prime}, N^{\prime}$-ethylenediamine tetraacetic acid; GTP, guanosine triphosphate; HEPES, 4-(2-hydroxyethyl)-1-piperazine ethanesulfonic acid; IPTG, isopropyl- $\beta$-D-thiogalactoside; L30a, Lactobacillus 30a; LDC, lysine decarboxylase; ODC, ornithine decarboxylase; PEG3350, polyethylene glycol with molecular weight of 3350; PLP, pyridoxal-5'-phosphate; SDS-PAGE, sodium dodecyl sulfate-polyacrylamide gel electrophoresis

\section{Introduction}

Ornithine decarboxylase (ODC) is a PLP-dependent enzyme which catalyzes the conversion of ornithine to putrescine. This is the initial and rate-limiting step in the synthesis of polyamines. Polyamines are necessary components of nearly all cells, from bacterial cells to those of plants and humans. Regulation of ODC activity occurs in the cell at the levels of transcription, translation and protein activity. Biosynthetic ODC from Escherichia coli is activated by ATP, GTP, CTP and UTP at millimolar concentrations (Anagnostopoulos et al., 1992), while rat-brain ODC is activated by GTP at micromolar levels (Kilpelainen \& Hietala, 1994).
Received 5 April 1999

Accepted 8 September 1999

PDB Reference: Gly121Tyr L30a ODC, 1c4k. 
Many bacteria contain both constitutive and inducible forms of amino-acid decarboxylases. Lactobacilli induce ODC under low-pH conditions in rich media to compensate for the $\mathrm{pH}$ drop associated with lactic acid production (Gale, 1946). Inducible ODC from Lactobacillus 30a (L30a) is a dodecamer with monomers of $83 \mathrm{kDa}$ and 730 amino acids (Hackert et al., 1994). It exhibits maximal activity at $\mathrm{pH} 5.8$ and the activity is lost above $\mathrm{pH} 7.0$, where the enzyme dissociates into dimers (Oliveira et al., 1997). At its pH optimum, the enzyme is not activated by nucleotides (Guirard \& Snell, 1980). At higher $\mathrm{pH}$ values, the enzyme can be reactivated by micromolar quantities of GTP, dGTP and GDP, but not by ATP, CTP or UTP. However, the dimer is not reassociated into dodecamers (Oliveira et al., 1997). Interestingly, the dodecamer binds two independent GTP molecules per dimer at $\mathrm{pH}$ 5.8. Half-site binding is observed at $\mathrm{pH} 8.0$, with the dimer binding only one GTP molecule tightly. The possibility of a conformational change in the dimer upon binding of the first GTP which is detrimental to the binding of a second at a neighboring site has been suggested (Oliveira et al., 1997).

The three-dimensional structure of inducible L30a ODC has been previously determined at $\mathrm{pH} 5.8$ in the absence of GTP (Momany, Ernst et al., 1995). The enzyme is 'doughnut shaped', with outer and inner diameters of 210 and $50 \AA$, respectively, and a height of $95 \AA$. It is a dodecamer assembled from six homodimers arranged with 622 point-group symmetry. The homodimers have an ellipsoidal core and two protruding 'wing' domains, one from each monomer, pointing into the central cavity region. Each monomer is arranged in five sequential folding units: wing (residues 1-107), linker (residues 108-160), PLP-binding (residues 161-411), specificity (residues 411-569) and C-terminal (residues 570730) domains. Wing and linker domains of adjacent dimers interact, stabilizing the dodecamer. Furthermore, the linker domain from one subunit forms part of the neighboring subunit's PLP-binding site and helps to stabilize the dimer. The PLP-binding domain includes a seven-stranded $\alpha / \beta$ fold, with the cofactor binding at the $\mathrm{C}$-terminal of a $\beta$-strand to Lys355 through a Schiff base in a manner similar to that seen in AAT (Ford et al., 1980; Kamitori et al., 1988). The fourth domain is the specificity domain, containing residues necessary for substrate recognition, as shown by sitedirected mutagenesis (Zhang \& Hackert, unpublished work). The C-terminal domain forms a ridge containing one wall of a $27 \AA$ deep channel at the dimer interface leading to the active site.

Four groups of PLP-dependent decarboxylases have been identified on the basis of sequence analysis (Sandmeier et al., 1994) and three of these four groups (groups I-III) are believed to utilize this type of PLP-binding motif (Momany, Ghosh et al., 1995). Group IV decarboxylases, such as mouse ODC, utilize an $\alpha / \beta$ barrel motif for binding the PLP cofactor (Kern et al., 1999), similar to that observed for ALR (Shaw et al., 1997). Of the four major fold types observed for PLPdependent enzymes (Grishin et al., 1995; Jansonius, 1998), the bacterial ODC belongs to the AAT family, while the eukaryotic ODCs are members of the ALR family.
A modified ODC was engineered using site-directed mutagenesis (Gopal, 1997) in which Gly121, which is buried in the dimer-dimer interface of the dodecamer, was replaced by Tyr. This prevented association of dimers into dodecamers. Size-exclusion chromatography, electron microscopy and light-scattering experiments showed that the Gly121Tyr ODC was a dimer. Unexpectedly, the dimers were active over the same $\mathrm{pH}$ range as the native enzyme, demonstrating that ODC activity was not dependent on the dodecameric state of the enzyme. Furthermore, the Gly121Tyr dimers were also activated by millimolar quantities of GTP at $\mathrm{pH}>$ 7.0. However, Scatchard plot analysis revealed two independent GTPbinding sites per dimer at both low and high $\mathrm{pH}$ (5.8 and 8.0).

This paper reports the structural analysis of the Gly121Tyr dimeric ODC at pH 7.0 in the presence of GTP. We undertook this structural analysis for the following reasons. Firstly, it was of interest to investigate the structural similarities and differences between modified (free) and native (associated) dimers resulting from the differences in the dimer-dimer interactions in the two structures. As expected, the modified and native dimers crystallize in different space groups and have different crystalline environments. In the native structure, the interactions between symmetry-related dimers form the dodecamer. In the mutant structure, dimer interactions only contribute to crystal cohesion. Secondly, it was of interest to compare the active sites of the two enzymes in order to determine whether any changes occurred in the transition from the dimeric to the dodecameric state. Thirdly, even though GTP binding to native ODC has been observed previously and briefly communicated (Oliveira et al., 1997), the resolution of the analysis $(3.8 \AA)$ was too low to analyze the interactions between GTP and the enzyme. The higher resolution of the present analysis allows us to analyze and discuss the various interactions involved in GTP binding in more detail.

\section{Experimental procedures}

\subsection{Protein preparation}

61 of $2 \times Y T$ media containing ampicillin were inoculated with bacteria HMS174/DE3 transformed with pET 21a with the mutant $L 30 \mathrm{a} o d c$ gene. The cells grew at $310 \mathrm{~K}$ for $3 \mathrm{~h}$ after induction with $0.5 \mathrm{~m} M$ isopropyl- $\beta$-D-thiogalactoside (IPTG). They were pelleted by centrifugation and stored frozen at $253 \mathrm{~K}$.

The frozen cells were resuspended in $50 \mathrm{~m} M$ potassium phosphate $\mathrm{pH} 6.5$ with $1 \mathrm{~m} M$ EDTA and $1 \mathrm{~m} M$ DTT and were subsequently broken using a French press at $110 \mathrm{MPa}$. The mutant protein was purified following the procedure of Guirard \& Snell (1980). The broken cells were centrifuged and both pellet and supenatant were assayed for ODC activity using a Gilson differential respirometer. The active soluble fraction was purified by column chromatography using DEAE cellulose and aminopentyl agarose columns. The fractions were assayed to identify the peak containing the mutant ODC protein activity. The quality of the purification was verified with SDS-PAGE and isoelectric focusing. 
Table 1

Summary of X-ray diffraction data collection.

\begin{tabular}{ll}
\hline Space group & $P 3_{2} 21$ \\
Unit-cell parameters $(\AA)$ & $a=111.8, c=135.9$ \\
Resolution $(\AA)$ & $50-2.7$ \\
Number of unique reflections & 26013 \\
Redundancy & 2.8 \\
Completeness $(\%)$ & 94.3 \\
Reflections with $I / \sigma<1(\%)$ & 9.6 \\
$R_{\text {sym }}^{\dagger}$ & 0.069
\end{tabular}

$\dagger R_{\text {sym }}=\sum\left|I_{\text {avg }}-I_{\text {obs }}\right| / \sum I_{\text {avg }}$, where $I$ is the average (avg) or the observed (obs) intensity of the reflection.

\subsection{Crystallization}

The purified protein was dialyzed against $100 \mathrm{~m} M$ sodium citrate- $\mathrm{HCl} \mathrm{pH} 6.5$ with $1.0 \mathrm{~m} M$ DTT, $0.2 \mathrm{~m} M$ PLP, $0.02 \%$ sodium azide and $0.5 \mathrm{~m} M$ EDTA, concentrated to $20 \mathrm{mg} \mathrm{ml}^{-1}$ using a Centricon-50 concentrator (Amicon) and mixed with GTP in a molar ratio of 1:20. Crystals were grown at $293 \mathrm{~K}$ by the sitting-drop method from reservoirs containing 30\% PEG 3350, $200 \mathrm{~m} M$ ammonium acetate and $100 \mathrm{~m} M$ Na HEPES pH 7.0. The drops consisted of equal volumes of reservoir and protein solution. The crystals have a cubic shape and a yellow color and belong to space group $P 3_{2} 21$, with unit-cell parameters $a=111.8, c=135.9 \AA$ and one monomer in the asymmetric unit. A $0.5 \mathrm{~mm}$ crystal was used for this study.

\subsection{Data measurement}

X-ray diffraction data were measured at $295 \mathrm{~K}$ using an R-AXIS IV image plate installed on a Rigaku RU200H rotating-anode $\mathrm{X}$-ray generator operated at $50 \mathrm{kV}$ and $100 \mathrm{~mA}$ with Molecular Structure Corporation mirrors. Oscillations were measured for $25 \mathrm{~min}$ each in $1.2^{\circ}$ intervals of $\varphi$. The crystal-to-detector distance was $160.0 \mathrm{~mm}$. The data were processed with the $H K L$ software package (Otwinowski
Table 2

Final refinement statistics.

\begin{tabular}{ll}
\hline Resolution range $(\AA)$ & $7.0-2.7$ \\
Number of reflections $(F>2 \sigma)$ & 24164 \\
Number of protein, PLP and GTP atoms & 5873 \\
Number of waters & 250 \\
$R(90 \%$ of data) & 0.212 \\
$R_{\text {free }}(10 \%$ of data) & 0.281 \\
$R \dagger($ all data) & 0.211 \\
R.m.s. deviations from ideal geometry & \\
$\quad$ Bond lengths $(\AA)$ & 0.013 \\
$\quad$ Bond angles $\left({ }^{\circ}\right)$ & 1.8 \\
$\quad$ Impropers $\left({ }^{\circ}\right)$ & 1.6 \\
Average thermal factors $\left(\AA^{2}\right)$ & \\
All atoms & 40.6 \\
$\quad$ Protein & 40.2 \\
GTP & 37.0 \\
PLP & 21.8 \\
$\quad$ Waters & 47.0 \\
Ramachandran plot & \\
$\quad$ Residues in normally allowed regions & 87.9 \\
$\quad$ (other than Gly and Pro) $(\%)$ & \\
$\quad$ Residues in disallowed regions $(\%)$ & 0 \\
\hline
\end{tabular}

$\dagger$ A few minor adjustments were made to the model before the final cycle including all data.

\& Minor, 1997). A summary of the X-ray data-collection statistics is presented in Table 1.

\subsection{Structure solution and refinement}

The structure was determined by molecular replacement using one of the monomers $(A)$ in the asymmetric unit of native ODC as the probe (PDB entry 1ord; Momany, Ernst et al., 1995). The calculations were carried out using $X$-PLOR 3.1 (Brünger, 1988, 1990, 1993b), data with $F>4 \sigma$ in the resolution range 4.0-15.0 $\AA$ and a shell of integration in the rotation function of 5.0-24.0 .

The model was refined by molecular-dynamics techniques (Brünger, 1988) using $X$-PLOR 3.1 and later $X$-PLOR 3.8 (Brünger, 1993b). The data were randomly divided in two sets; a working set composed of $90 \%$ of the data and a test set composed of the remaining $10 \%$ for cross-validation purposes (Brünger, 1993a). The slow-cool annealing protocol was used for simulating annealing, with a dynamics temperature starting at $3000 \mathrm{~K}$. The refinement was carried out with data in the resolution range 2.7-7.0 $\AA$ and $F>2 \sigma$, with weights set to half the weight recommended by the check stage and the protein parameters of Engh \& Huber (1991). The parameters for PLP and GTP were determined using the program XPLO2D (Kleywegt \& Jones, 1997). Only one group temperature factor was refined per residue based on comparisons of $R_{\text {free }}$ values. The refinement alternated between manual building and rebuilding of the model using the program $O$ (Jones et al., 1991). At the completion of the protein refinement, water molecules were assigned to density if they corresponded to peaks $>2.5 \sigma$ in the $F_{o}-F_{c}$ difference electron-
Figure 1

A stereoview of $2 F_{o}-F_{c}$ electron density around GTP. Contours are at 1.35o. Figs. 1, 2, 4 and 5 were drawn using $O$ (Jones et al., 1991). 

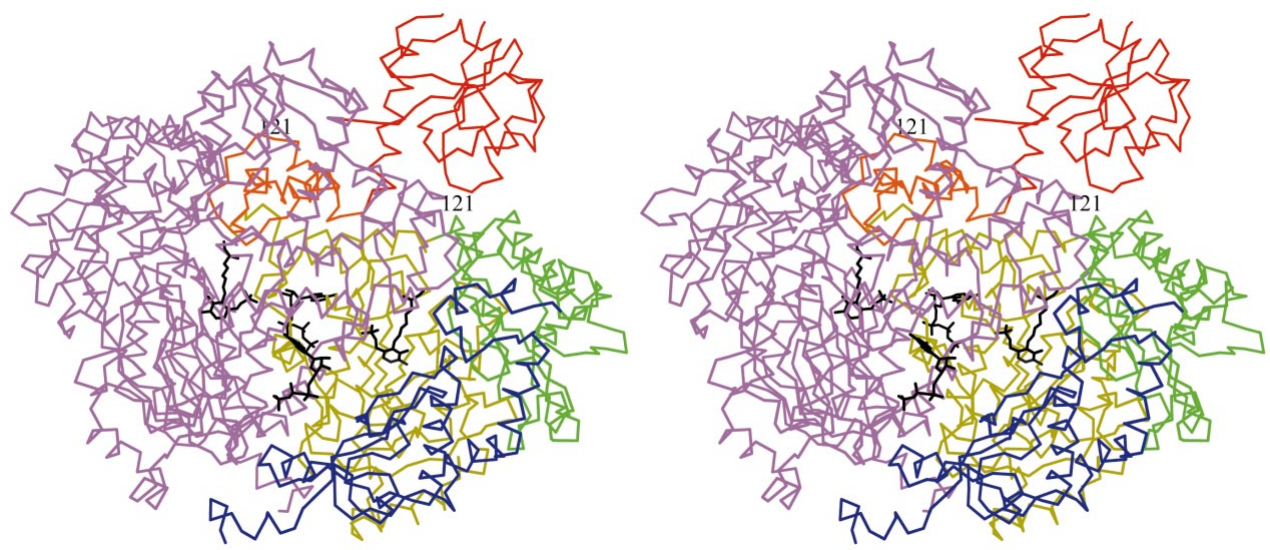

Figure 2

Stereo $\mathrm{C}^{\alpha}$ backbone trace of the Gly121Tyr dimer. One monomer is shown in magenta and the other has its sequential domains colored as follows: wing (red), linker (orange), PLP-binding (yellow), specificity (green) and C-terminal (blue). The GTP effectors (black) are bound at the surface of each monomer along the subunit interface at the entrance to the deep channel leading to the active site containing the PLP cofactor (black). The twofold crystallographic axis relating the two subunits is nearly vertical in this view. The mutation site (121) is also indicated and is part of a strand that runs from the surface of the subunit to above the cofactor site.

density map, positive electron density in the $2 F_{o}-F_{c}$ electrondensity maps and if they had appropriate hydrogen-bonding interactions.

\subsection{Model analysis}

Structural superpositions were carried out with the program ALIGN (Satow et al., 1986; Cohen, 1997). Local superpositions were carried out with $O$ (Jones et al., 1991). Hydrogen bonds were identified by a donor-acceptor distance $<3.5 \AA$ and by angles $X$-donor $\cdots$ acceptor and donor $\cdot$ acceptor $-Y>90^{\circ}$. A distance of $3.5 \AA$ was also used as a cut-off in the identification of salt bridges. Two atoms were considered to make a contact if their separation was less than $4.0 \AA$.

\section{Results and discussion}

\subsection{Structure solution and refinement}

The correct solution was strongly indicated by the molecular-replacement searches. It corresponded to the top peak in both the Patterson correlation refinement and translation search; the signal-to-noise ratios, defined as the ratios of the correct peak to the top noise peak, were 3.92 in the Patterson correlation refinement and 1.69 in the translation search. A rigid-body refinement resulted in $R=0.402$ and $R_{\text {free }}=0.412$ for data with $F>2 \sigma$ in the resolution range 2.7-7.0 ̊.

The ODC Gly121Tyr-GTP model presented here consists of 5873 non-H atoms, representing 728 out of the 730 aminoacid residues of the protein, one cofactor, one GTP and 250 waters. Loop residues Asp84 and Lys85 were not included in the final model. The final map, contoured at $0.9 \sigma$, shows continuous density for most main-chain and side-chain atoms, with minor discontinuities in the main chain at residues Asp19, Gln563 and Ile579 and with weak or absent electron density for a few side chains, mostly Lys, Arg, Glu, Asp and Gln. There is also weak and fragmented electron density for the first $\mathrm{N}$-terminal and the last two C-terminal residues, as well as for residues Glu83-Ala88. There is only weak density for the side chain of the modified residue Tyr121, presumably owing to disorder since it is now a surface residue with no intermolecular interactions. The final crystallographic $R$ factor, including all reflections with $F>2 \sigma$ in the $2.7-7.0 \AA$ resolution shell is 0.211 . A summary of the model refinement statistics is presented in Table 2. A $2 F_{o}-F_{c}$ electron-density map for the GTP is shown in Fig. 1.

\subsection{Description of the structure}

The $\mathrm{C}^{\alpha}$ backbone of the Gly121Tyr dimer viewed into its active site is shown in Fig. 2. The two subunits are related by a crystallographic twofold axis, while the subunits of the dimer in the P6 crystals of the native dodecameric ODC (Momany, Ernst et al., 1995) were related by a non-crystallographic twofold axis. The PLP cofactor, covalently bonded via a Schiff base to Lys355 of one subunit, is located in the active site at the bottom of the $27 \AA$ deep channel formed at the dimer interface. The GTP effector, bound via non-covalent interactions primarily involving the other subunit, is located at one entrance of this channel. The position of the modified residue, Gly121, is indicated in Fig. 2 by a label. Gly121 in the native enzyme is in a hydrophobic cavity formed by the side chains of Val90"' 1 and the aliphatic parts of the side chains of Arg93" and Glu94"' of the wing domain from a neighboring dimer in the dodecamer. The mutation was designed to disrupt these contacts and prevent dodecamer formation. In the following sections, the Gly121Tyr dimer is compared with the native ODC dimer (Momany, Ernst et al., 1995). Detailed descriptions of the active and GTP-binding sites are given.

\subsection{Comparison with native ODC}

The Gly121Tyr and native ODC dimers are closely similar. The major differences between the two structures occur in the wing domains, which show both rigid-body movements from

\footnotetext{
${ }^{1}$ Primes in residue numbers indicate the twofold related monomer. Double primes indicate a symmetry-related dimer in the present structure. Triple primes indicate another dimer in dodecameric ODC.
} 
the native (Fig. 3) as well as larger overall r.m.s. deviations between $C^{\alpha}$ atoms relative to the rest of the molecule. Superposition of the $\mathrm{C}^{\alpha}$ positions of domains $2-5$ of one subunit from each dimer results in an r.m.s. deviation of $0.44 \AA$ for residues 108-730 used in the superposition. Subsequent superposition of the wing domains of these subunits, residues $1-107$, requires an additional rotation of $5.4^{\circ}$ and translation by $0.28 \AA$. The resulting r.m.s. deviation for $\mathrm{C}^{\alpha}$ atoms of the superimposed wing domains is $0.71 \AA$. Since the intradimer twofold axes are also tilted relative to each other by $1.5^{\circ}$, additional shifts are needed to superimpose the wing domain and domains $2-5$ of the second subunit in the dimer. Thus, superposition of the wing domain and of domains 2-5 of the other subunit requires larger rotations and translations of $6.3^{\circ}$ and $0.90 \AA$, respectively, for the wing and of $1.5^{\circ}$ and $0.10 \AA$, respectively, for domains $2-5$. It is possible that the observed differences in the spatial disposition of the domains relative to each other from the native structure suggest an inherent flexibility in the ODC monomer, with the wing domains capable of changing their orientation, adapting to their environment in the crystal, solution and in the native dodecameric structure. Some flexibility is also observed in the association of the monomers that form the dimer. It is worth noting in this regard that $E$. coli arginine and lysine decarboxylases have very similar subunit sizes and show significant sequence homology with L30a ODC in the linker through $\mathrm{C}$ terminal domains, but less homology in the wing domains. Interestingly, the $E$. coli ADC (Boeker \& Snell, 1968) and LDC (Sabo \& Fischer, 1974) are observed to form stable decamers rather than the dodecamer observed for $L 30$ a ODC.

There are only a few residues significantly differing in their $\mathrm{C}^{\alpha}$ positions after superposition. The differences appear to be related either to crystal packing or to interactions between dimers in the dodecamer. Pro505 and Glu506 on the surface of the dimer shift by 2.77 and $1.64 \AA$ from the dodecameric structure, respectively, apparently to avoid short contacts with Ala70" of a symmetry-related dimer in the present structure. Also, the $\mathrm{C}^{\alpha}$ positions of Tyr121, which is on the surface of dimeric ODC, and Gly121, which is buried in the native enzyme, are displaced relative to each other by $1.85 \AA$. In the native enzyme, Gly121 must avoid short contacts with Glu94"' of the wing domain of the neighboring dimer in the dodecamer.

A few residues, particularly Lys, Arg and, less frequently, Glu and Gln on the surface of the monomer, also have different sidechain orientations in the two structures. Apart from the natural flexibility inherent in these long side chains, in most instances their crystalline environments also

Figure 4

Active site. Gly121Tyr (C black, O red, $\mathrm{N}$ blue) and native (yellow) ODCs locally superimposed. Thin dashed lines correspond to hydrogen bonds in the present structure. The lengths of hydrogen bonds and

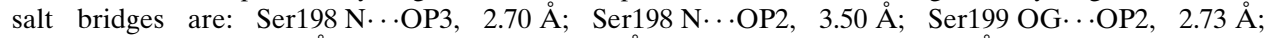

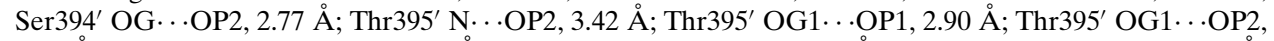

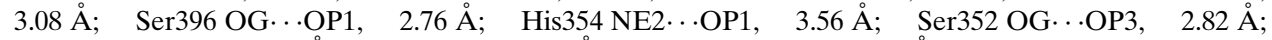

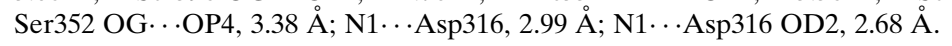


differ. Of special interest are Lys190 and Lys374 in the GTPbinding pocket, the side-chain conformations of which change from those observed in the native structure in order to interact more favorably with the GTP phosphates. In addition, the side chain of Lys190 reorients, since in the native structure it occupies a portion of the space used for the guanine base in the GTP-binding pocket.

Phe77 and His78 of the wing domain have different sidechain conformations from the native and make different

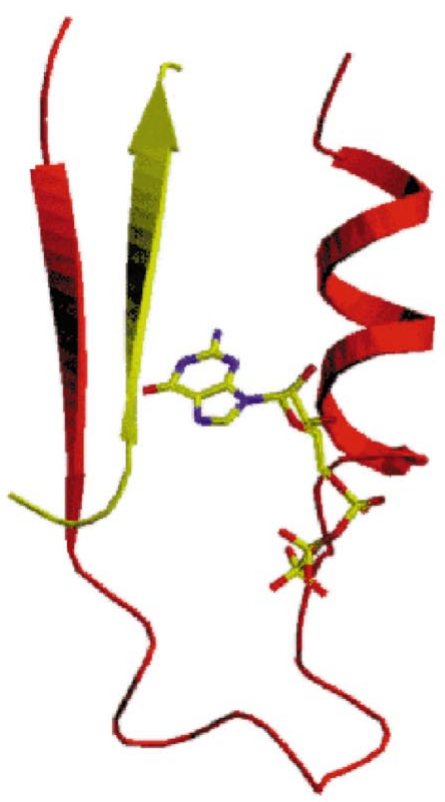

(a)
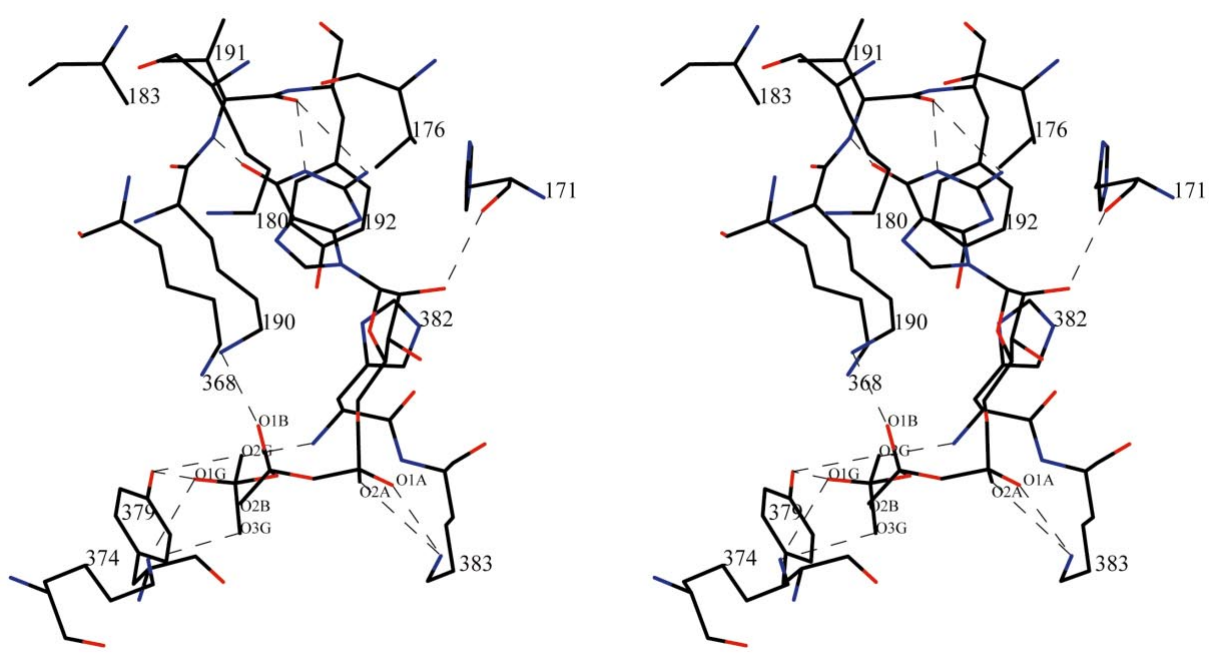

(b)

Figure 5

(a) Ribbon drawing of the topology of the GTP-binding site in Gly121Tyr ODC. The $\beta$-strand (residues 364-369) and $\alpha$-helix (residues 384-392) which define the loop are in red. The $\beta$-strand (residues 189194) that binds the guanine base is in yellow. The nucleotide is also shown. (b) A stereoview of the interactions between GTP and protein. Hydrogen bonds and salt bridges are shown by thin dashed lines. The hydrophobic site which accommodates the guanine base is also shown. The lengths of

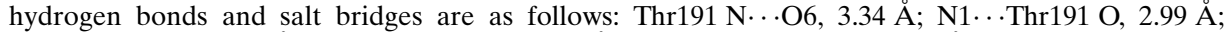

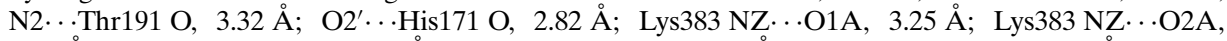

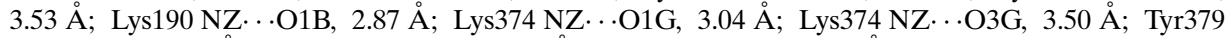

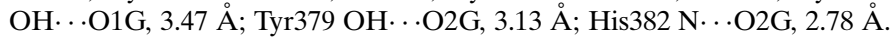

be protonated. This is supported by the fact that in the native structure at $\mathrm{pH} 5.8$ both ND1 and NE2 are involved in salt bridges and hydrogen bonds; ND1 makes an intrasubunit salt bridge with Glu94 OE1 (3.49 $)$ ) and NE2 makes an interdimer hydrogen bond with His527"' $\mathrm{O}(3.40 \AA)$. The $\mathrm{p} K_{a}$ of His 78 would be expected to be elevated by this environment. It is interesting that His78 appears not to be protonated in the present structure at $\mathrm{pH}$ 7.0. One of its imidazole $\mathrm{N}$ atoms, ND1, hydrogen bonds with Asp457" OD2 (2.65 $)$ ) of a symmetry-related dimer, but the second $\mathrm{N}$ atom is not involved in any such interactions. The conformational change observed for Phe77 is probably the result of the conformational change of His78, as this residue is deprotonated at higher $\mathrm{pH}$, disrupting the salt-bridge interaction to Glu94.

\subsection{The active site}

The refined structure has continuous density from the pyridoxal ring to Lys355, confirming the presence of the internal aldimine form of the cofactor. Fig. 4 shows a comparison of the active sites in the Gly121Tyr dimer and native ODCs (Momany, Ernst et al., 1995). The two active sites are closely similar. The phosphate charge of PLP is stabilized by a salt bridge with His 354 and by the positive dipole arising from the $\mathrm{N}$-terminal end of the $\alpha$-helix formed by residues Ser198-Leu209 (not shown in the figure). Furthermore, there are five serines and one threonine (Ser198, Ser199, Ser352, Ser394', Thr395' and Ser396') within hydrogen-bonding distance of the phosphate $\mathrm{O}$ atoms. The pyridinium $\mathrm{N}$ atom forms salt bridges with Asp316; the pyridinium ring is sandwiched between the side chains of His223 and Ala318, interacting with the imidazole of His223 in a parallel manner. Additionally, the indole of Trp319 makes an edge-on interaction with the cofactor (Burley \& Petsko, 1985). The side chain of Glu532, which modeling studies suggested to be the substrate-specificity residue interacting with $\mathrm{NE}$ of the substrate L-ornithine in the active site (Momany, Ernst et al., 1995), also has the same orientation as observed in the native structure. Residues Arg120'-Phe125' of the neighboring monomer fill in the active site. The $\mathrm{C}^{\alpha}$ of $\operatorname{Tyr} 121^{\prime}$ is displaced from the corresponding $\mathrm{C}^{\alpha}$ position of Gly121' in the native ODC by $1.85 \AA$.

The similarity of the active sites of the two enzymes is consistent with the observation that the activity of ODC does not depend on its dodecameric state (Gopal, 1997). Furthermore, it shows that GTP binding does not cause any significant conformational changes in the 
active site of the enzyme. This is consistent with biochemical observations (Gopal, 1997) that Gly121Tyr ODC is equally active in the presence of GTP over the $\mathrm{pH}$ range 5.5-8.5 and in the absence of GTP over the $\mathrm{pH}$ range 5.5-7.5. It is possible that inactive ODC or Gly121Tyr ODC at $\mathrm{pH}>7.5$ and in the absence of GTP has a different conformation from that seen in the present structure.

\subsection{The GTP-binding site}

Perhaps most importantly, the Gly121Tyr dimer structure has enabled a higher resolution model of the GTP effector binding site in bacterial ODC. The overall mode of GTP binding to Gly121Tyr ODC (Fig. 5a) appears similar to that observed at low $(3.8 \AA)$ resolution reported in the kinetic characterization of GTP effects (Oliveira et al., 1997). The higher resolution of the present analysis allows an evaluation of the interactions involved in the binding.

The allosteric site is situated on the surface of one subunit, at the entrance to a deep active-site channel about $27 \AA$ from the cofactor site (Fig. 2). This site is near a dimer interface in native ODC. This is a unique type of GTP-binding site (Oliveira et al., 1997). In the Ras superfamily of GTPases, elongation factor $\mathrm{Tu}$ and the $\mathrm{G}$ proteins of signal transduction, the GTP-binding site is typically located near the C-terminal end of a parallel $\beta$-strand, with the second phosphate typically positioned near the positive dipole at the $\mathrm{N}$-terminal end of an $\alpha$-helix (Pai et al., 1989; Coleman et al., 1994). ODC contains a large seven-stranded $\beta$-sheet. Six of these seven strands run towards the center of the dimer, with the cofactor-binding site at the $\mathrm{C}$-terminal end of one of those strands. The remaining strand runs antiparallel to the rest of the sheet towards the surface of the dimer, where it makes a short loop before returning via a short $\alpha$-helix. The phosphates of GTP bind near the N-terminal end of this short helix (Fig. 5a). The presence of a single $\beta$-strand and a return helix appear to be the only structural elements in common with the other GTPbinding proteins. The guanine base is positioned in a very different manner, held by a hydrogen bond to a $\beta$-strand that runs antiparallel to the $\beta$-strand that makes the loop (Fig. 5a).

The GTP environment is shown in Fig. 5(b). The guanine base fits in a hydrophobic pocket formed by the side chains of His171, Val176, the aliphatic parts of Lys180 and Lys190, Tyr192, His382 and Ala183, and makes close contacts with these residues. The specificity for GTP is apparent in that hydrogen-bond donors and acceptors are specific for guanine. O6 accepts a hydrogen bond from the amide $\mathrm{N}$ of Thr191, and both $\mathrm{N} 1$ and the amino $\mathrm{N} 2$ donate a hydrogen bond to the carbonyl $\mathrm{O}$ atom of Thr191. Replacement of the guanine base with adenine, uracil and cytosine would disrupt the favorable guanine-specific base-protein interactions seen in Fig. 5(b) and explains why ATP, UTP and GTP are not activators for L30a ODC activity. Few interactions are made with the ribose sugar of the nucleotide; there is a hydrogen bond involving $\mathrm{O} 2^{\prime}$ and the carbonyl $\mathrm{O}$ atom of His171. However, there are extensive ion pairs and hydrogen-bonding interactions observed for the triphosphate. Charge neutralization occurs through interactions with Lys190, Lys374 and Lys383. Lys368 and Lys180 are also nearby but further from the phosphate. Lys190 and Lys374 change their side-chain conformations in order to interact more effectively with the GTP phosphate O atoms and, in the case of Lys190, to make room for the guanine base. The terminal phosphate is not only ion-paired with Lys374, but further stabilized by hydrogen bonds from the hydroxyl group of Tyr379 and the amide $\mathrm{N}$ atoms of His382. These interactions probably account for the stronger binding of GTP compared with GDP.

The mechanism of the GTP effect is not understood other than that the binding of GTP extends the $\mathrm{pH}$ profile for activity by lowering the $K_{M}$ for the substrate. In native ODC (Oliveira et al., 1997), it was postulated that GTP binding would cause a conformational change to further isolate the active site from solvent, which might produce a shift in the $\mathrm{p} K_{a}$ of an essential residue from 6.7 to 8.2. However, we do not observe a conformational change from the native in the present structure. In the Gly121Tyr dimeric form of ODC, the loss of activity at high $\mathrm{pH}$ is accompanied by a very sharp slope suggestive of the loss of several protons. Since there are several lysines involved in the binding of GTP, it seems reasonable that GTP binding might be lost as the $\mathrm{pH}$ increases to the point where these lysines lose their positive charge.

Work was supported in part by NIH grant GM 30105 and a grant from the Foundation for Research to MLH.

\section{References}

Anagnostopoulos, C., Choli, T. \& Kyriakidis, D. A. (1992). Biochem. Intl, 27, 991-1000.

Boeker, E. A. \& Snell, E. E. (1968). J. Biol. Chem. 243, 1678-1684. Brünger, A. T. (1988). J. Mol. Biol. 208, 803-816.

Brünger, A. T. (1990). Acta Cryst. A46, 46-57.

Brünger, A. T. (1993a). Acta Cryst. D49, 24-36.

Brünger, A. T. (1993b). X-PLOR Manual, Version 3.1. Yale University, New Haven, Connecticut, USA.

Burley, S. K. \& Petsko, G. A. (1985). Science, 229, 23-28.

Cohen, G. H. (1997). J. Appl. Cryst. 30, 1160-1161.

Coleman, D. E., Berghuis, A. M., Lee, E., Linder, M. E., Gilman, A. G. \& Sprang, S. R. (1994). Science, 265, 1405-1412.

Engh, R. A. \& Huber, R. (1991). Acta Cryst. A47, 392-400.

Esnouf, R. M. (1997). J. Mol. Graph. 15, 132-134.

Ford, G. C., Eichele, G. \& Jansonius, J. N. (1980). Proc. Natl Acad. Sci. USA, 77, 2559-2563.

Gale, E. F. (1946). Adv. Enzymol. 6, 1-32.

Gopal, R. (1997). MA thesis, University of Texas, Austin, Texas, USA. Grishin, N. V., Phillips, M. A. \& Goldsmith, E. J. (1995). Protein Sci. 4, 1291-1304.

Guirard, B. M. \& Snell, E. E. (1980). J. Biol. Chem. 255, 5960-5964.

Hackert, M. L., Carroll, D. W., Davidson, L., Kim, S.-O., Momany, C., Vaaler, G. L. \& Zhang, L. (1994). J. Bacteriol. 176, 7391-7394.

Jansonius, J. N. (1998). Curr. Opin. Struct. Biol. 8, 759-769.

Jones, T. A., Zou, J. Y., Cowan, S. W. \& Kjeldgaard, M. (1991). Acta Cryst. A47, 110-119.

Kamitori, S., Hirotsu, K., Higuchi, T., Kondo, K., Inoue, K., Kuramitsu, S., Kagamigama, H., Higuchi, Y., Yasuoka, N., Kusunoki, M. \& Matsuura, Y. (1988). J. Biochem. (Tokyo), 104, 317-318.

Kern, A., Oliveira, M., Coffino, P. \& Hackert, M. L. (1999). Structure, 7, 567-581. 
Kilpelainen, P. T. \& Hietala, O. A. (1994). Biochem. J. 300, 577-582. Kleywegt, G. J. \& Jones, T. A. (1997). Methods Enzymol. 277, 208-230.

Kraulis, P. J. (1991). J. Appl. Cryst. 24, 946-950.

Momany, C., Ernst, S., Ghosh, R., Chang, N.-L. \& Hackert, M. L. (1995). J. Mol. Biol. 252, 643-655.

Momany, C., Ghosh, R. \& Hackert, M. L. (1995). Protein Sci. 4, 849-854.

Oliveira, M. A., Carroll, D., Davidson, L., Momany, C. \& Hackert, M. L. (1997). Biochemistry, 36, 16147-16154.
Otwinowski, Z. \& Minor, W. (1997). Methods Enzymol. 276, 307-326. Pai, E. F., Kabsch, W., Krengel, U., Holmes, K. C., John, J. \& Wittinghofer, A. (1989). Science, 341, 209-214.

Sabo, D. L. \& Fischer, E. H. (1974). Biochemistry, 13, 670-676.

Sandmeier, E., Hale, T. I. \& Christen, P. (1994). Eur. J. Biochem. 221, 997-1002.

Satow, Y., Cohen, G. H., Padlan, E. A. \& Davies, D. R. (1986). J. Mol. Biol. 190, 593-604.

Shaw, J. P., Petsko, G. A. \& Ringe, D. (1997). Biochemistry, 36, 1329-1342. 